\title{
Discrete-Time Networked Dynamic Systems
}

\author{
MAGDI S. MAHMOUD \\ Distributed Control Research Lab \\ Systems Engineering Department, KFUPM \\ P. O. Box 5067, Dhahran 31261 \\ SAUDI ARABIA
}

\begin{abstract}
In this paper, we provides a general framework for the analysis of a class of linear discrete-time networked dynamic systems (DNDS). We focus our attention on DNDS where the underlying connection topology couples the agents at their outputs. A distinction is made between DNDS with homogeneous agent dynamics and DNDS with heterogeneous agent dynamics. It is emphasized that developing efficient solution methods for the design of such systems involve connecting and interpreting results from graph theory and convex optimization in a systems-theoreticcontext.
\end{abstract}

Key-Words: Discrete-time networked dynamic systems (DNDS), Multiagent systems, homogeneous agent dynamics, heterogeneousagentdynamics.

Received: November 23, 2019. Revised: April 21, 2020. Accepted: May 9, 2020. Published: May 22, 2020.

\section{Introduction}

Networked dynamic systems (NDS) are a collection of multiple dynamic systems that are coupled together through a network. These types of systems are found in a range of applications that involve, for example, the coordination of multiple space, air, and land vehicles [1], [2], [3], [4]. Studying system theoretic notions from the perspective of the underlying topology can lead to interpretations that explicitly characterize the effects of the network on the behavior of the system.

For linear and time-invariant systems, all the essential systems theoretic properties can be derived from the quadruple system matrices $(A, B, C, D)$. When considering multiagent systems, the underlying connection topology, $\mathcal{G}$, can typically be embedded into the system matrices. It is then enlightening to consider how certain properties of the system explicitly depend on that topology. Therefore, when studying linear NDS, one should consider the quintuple $(A, B, C, D, \mathcal{G})$ and explicitly describe the dependance of the underlying topology on the system properties. Recent examples of such network-centric analysis include relating closed-loop stability properties of NDS to the spectral properties of the graph Laplacian [5], relating controllability in consensus seeking systems to graph symmetry [6], and graph-centric observability properties of relative sensing NDS [7]. In this work we focus on a class of linear NDS where the underlying connection topology couples the agents at their outputs. Such systems are prevalent in formation flying applications where relative sensing is used to measure inter-agent distances [8].

The main contribution of this paper is a graphcentric characterization of the system $\mathcal{H}_{2}$ norm for both analysis and synthesis purposes. A distinction is made between NDS with homogeneous agent dynamics and NDS with heterogeneous agent dynamics. Although the homogenous case is actually a subset of the heterogeneous case, it is more illuminating to consider these cases separately due to the algebraic simplicity of the former case.

For the synthesis portion of this paper we consider two general design scenarios that can be akin to an inner-loop control design for an NDS. In the first case, we focus on the design of a local $\mathcal{H}_{2}$ controller for each agent when the underlying connection topology is given and fixed. In addition to satisfying local performance objectives (such as those typically found in $\mathcal{H}_{2}$ synthesis), the proposed synthesis procedure also satisfies a global NDS objective related to the underlying connection topology. A semi-definite program is derived as a solution method for this problem. The second synthesis objective focuses on the design of the connection topology that optimizes the $\mathcal{H}_{2}$ performance of the NDS. Topology design can be considered a problem in combinatorial optimization, which can be a prohibitively hard to solve when the number of agents is large. The results of this paper shows that the problem can be solved using Kruskal's minimum spanning tree algorithm. It should also be noted that the design of the underlying topology in the context of systems theoretic properties, such as the $\mathcal{H}_{2}$ norm, 
has received little attention in the literature.

\section{Algebraic Graphs Representation}

We make use of results from algebraic graph theory. The reader is referred to [9] for a detailed treatment of the subject and we present here only a minimal summary of relevant constructs and results. An undirected (simple) graph $\mathcal{G}$ is specified by a vertex set $\mathcal{V}$ and an edge set $\mathcal{E}$ whose elements characterize the incidence relation between distinct pairs of $\mathcal{V}$.

We make extensive use of the $|\mathcal{V}| \times|\mathcal{E}|$ incidence matrix, $\mathcal{E}(\mathcal{G})$, for a graph with arbitrary orientation. The columns of $\mathcal{E}(\mathcal{G})$ are indexed by the edges, and the $i^{\text {th }}$ row entry takes the value one if it is the initial node of the corresponding edge, negative one if it is the terminal node, and zero otherwise. The degree of vertex $i, d_{i}$, is the cardinality of the set of vertices adjacent to it. The diagonal matrix $\Delta(\mathcal{G})$ contains the degree of each vertex on its diagonal. The adjacency matrix, $A(\mathcal{G})$, is the symmetric $|\mathcal{V}| \times|\mathcal{V}|$ matrix with zero on the diagonal and one in the $i j^{\text {th }}$ position if node $i$ is adjacent to node $j$.

The (graph) Laplacian of $\mathcal{G}$,

$$
\mathcal{L}(\mathcal{G}):=\mathcal{E}(\mathcal{G}) \mathcal{E}(\mathcal{G})^{T}=\Delta(\mathcal{G})-A(\mathcal{G}),
$$

is a rank deficient positive semi-definite matrix.

\subsection{Notations}

In the sequel, the Euclidean norm is used for vectors. We use $W^{t}, W^{-1}$ and $\lambda(W)$ to denote, respectively, the transpose, the inverse and the eigenvalues of any square matrix $W$ and $W>0(W<0)$ stands for a symmetrical and positive- (negative-) definite matrix $W$. The n-dimensional Euclidean space and the space of bounded sequences are denoted by $\mathbb{R}^{n \times n}$ and $\ell_{2}$, respectively. The term $\mathcal{D}_{\text {int }}(0,1)$ denotes the interior of the unit disk with center at the origin and $\lambda\left(E_{o}, A_{o}\right)=\left\{z \mid \operatorname{det}\left(z E_{o}-A_{o}\right)=0\right\}$. Let $\mathrm{C}_{n, d}:=$ $\mathrm{C}\left([-d, 0], \Re^{n}\right)$ denotes the Banach space of continuous vector functions mapping the interval $[-d, 0]$ into $\left.\Re^{n}\right)$ and $x_{k+\alpha} \in \mathrm{C}_{n, d}, \alpha \in[-d, 0], k \geq 0$ denotes the function family defined on $[-d, 0]$ which is generated by $n$-dimensional real vector-valued function $x_{k}, k \in[-d, \infty)$. We use $\|$.$\| to represent the Eu-$ clidean norm for vectors and the spectral norm for matrices and $\|\psi\|_{c}:=\sup _{-d<d_{k}<0}\|\psi\|$ stands for the norm of function $\psi \in \mathrm{C}([-d, \overline{0}])$. Matrices, if their dimensions are not explicitly stated, are assumed to be compatible for algebraic operations. In symmetric block matrices or complex matrix expressions, we use the symbol $\bullet$ to represent a term that is induced by symmetry.
Sometimes, the arguments of a function will be omitted when no confusion can arise.

\subsection{Matrix Kronecker Products}

Some important results on the Kronecker product are presented hereafter. The Kronecker product of two matrices $A$ and $B$ is written as $A \otimes B$.

Theorem 1 ([10]): Let $A \in \Re^{m \times n}$ and $B \in \Re^{p \times q}$ each have a singular value decomposition of $A=$ $U_{A} \Sigma_{A} V_{A}^{T}$ and $B=U_{B} \Sigma_{B} V_{B}^{T}$. The singular value decomposition of the Kronecker product of $A$ and $B$ is then

$$
A \otimes B=\left(U_{A} \otimes U_{B}\right)\left(\Sigma_{A} \otimes \Sigma_{B}\right)\left(V_{A}^{T} \otimes V_{B}^{T}\right)
$$

Corollary 2 An immediate consequence of Theorem 1 is the following result on the matrix $2-$ norm, $\| A \otimes$ $B\left\|_{2}=\right\| A\left\|_{2}\right\| B \|_{2}$.

In the sequel, We also make extensive use of the following Kronecker product matrix multiplication property, $(A \otimes B)(C \otimes D)=(A C \otimes B D)$

\section{Networked Dynamic Systems}

In the sequel, we adopt the view that a networked dynamic systems (NDS) consists of two system layers. The first can be considered as the local agent layer corresponding to the dynamics of the individual agents in the ensemble. The second layer is a global NDS layer that represents the complete interconnected system. This section develops a general linear model for NDS that includes both the local and global layers.

\subsection{Problem Statement}

We will work with the following class of discrete networked dynamic systems (DNDS):

$$
\begin{aligned}
x_{i}(k+1) & =A_{i} x_{i}(k)+B_{i} u_{i}(k)+\Gamma_{i} \omega_{i}(k), \\
z_{i}(k) & =C_{i} x_{i}(k)+D_{i} u_{i}(k) \\
y_{i}(k) & =G_{i} x_{i}(k)
\end{aligned}
$$

Define the vertex set $\mathcal{N}=\{1, \ldots, N\}$. We use $\left\{A_{o}, \ldots, \Gamma_{o}\right\}$ to imply generic system matrices and $\left\{A_{o j}, \ldots, \Gamma_{o j}, j \in \mathcal{N}\right\}$ to represent the respective values at the vertices. In system (2), $x_{k} \in \Re^{n}$ is the state vector, $\omega_{k} \in \Re^{q}$ is the disturbance input which belongs to $\ell_{2}[0, \infty), y_{k} \in \Re^{p}$ is the measured output and $z_{k} \in \Re^{q}$ is the controlled output. The matrix $E_{o} \in \Re^{n \times n}$ may be singular; we assume that rank $E_{o}=r<n$. The matrices $A_{o} \in \Re^{n \times n}, B_{o} \in \Re^{n \times m}$, 
$G_{o} \in \Re^{q \times n}, \quad C_{o} \in \Re^{p \times n}, D_{o} \in \Re^{p \times n}, \quad \Gamma_{o} \in \Re^{n \times q}$ are real and known constant matrices. Henceforth, we use $\mathcal{S}:=\left\{\psi_{\mathbf{k}} \mid \psi_{\mathbf{k}} \in \mathcal{S}_{\mathbf{o}}\right.$, there exists a unique solution for system (2) on the interval $[0, \infty)$ for $\psi_{k}$ and $\mathcal{B}:=\left\{\psi_{\mathbf{k}} \mid \psi_{\mathbf{k}} \in \mathbf{C}_{\mathbf{n}, \mathbf{d}},\left\|\psi_{\mathbf{k}}\right\|_{\mathbf{c}} \delta, \delta>\mathbf{0}\right\}$

As we are focusing on the $\mathcal{H}_{2}$ properties of this system, we assume no feedforward term of the control $u_{i}(k)$ and no noises in the measurements (e.g. strictly proper system). Additionally, we assume a minimal realization for each agent with the outputs of each agent being compatible (e.g., system outputs correspond to the same physical quantity).

We denote the open-loop map from $\omega_{i}(k)$ to $y_{i}(k)$ as $T_{i}^{\omega \rightarrow y}$ and the closed-loop map from $\omega_{i}(k)$ to $z_{i}(k)$ as $T_{i}^{\omega \rightarrow z}$. The $\mathcal{H}_{2}$ synthesis problem for a local agent is to design a feedback controller of the form $u_{i}(k)=$ $L_{i} y_{i}(k)$ that minimizes the closed-loop system norm, $\left\|T_{i}^{\omega \rightarrow z}\right\|_{2}$

The parallel interconnection of all agents is described with the following state-space description of DNDS:

$$
\begin{aligned}
\mathbf{x}(k+1) & =\mathbf{A x}(k)+\mathbf{B u}(k)+\Gamma \omega(k), \\
\mathbf{z}(k) & =\mathbf{C x}(k)+\mathbf{D u}(k) \\
\mathbf{y}(k) & =\mathbf{G x}(k)
\end{aligned}
$$

with $\mathbf{x}, \mathbf{u}, \omega, \mathbf{z}, \mathbf{y}$ and $\omega$ denoting, respectively, the concatenated state vector, control vector, exogenous input vector, controlled vector, measured output vector and exogenous disturbance input at time $k$ of all the agents in the NDS. The matrices $\mathbf{A}, \mathbf{B}, \mathbf{C}, \mathbf{G}, \mathbf{D}$ and $\Gamma$ are the block diagonal aggregation of each agent's state-space matrices.

The global DNDS layer we examine for the duration of this paper is motivated by the relative sensing problem. The sensed output of the NDS is the vector $\mathbf{y}_{\mathcal{G}}(k)$ containing the relative state information of each agent and its neighbors. For example, the output sensed across an edge $e=(i, j)$ would be of the form $y_{i}(k)-y_{j}(k)$. This can be compactly written as

$$
\mathbf{y}_{\mathcal{G}}(k)=\left(\mathcal{E}(\mathcal{G})^{T} \otimes I\right) \mathbf{y}(k)
$$

The global layer is visualized in the block diagram in Fig. 1. When considering the analysis of the global layer, we are interested in studying the map from the agent's exogenous inputs to the NDS sensed output, which we denote by the operator $T_{h o m}^{\omega \rightarrow \mathcal{G}}$ homogeneous NDS, and $T_{h e t}^{\omega \rightarrow \mathcal{G}}$ for heterogeneous NDS. Using the above notations and the Kronecker properties outlined earlier, we can express the homogeneous and heterogeneous as

We identify two classes of DNDS in this wok:

1) homogeneous DNDS, where it is assumed that each dynamic agent in the NDS is described by

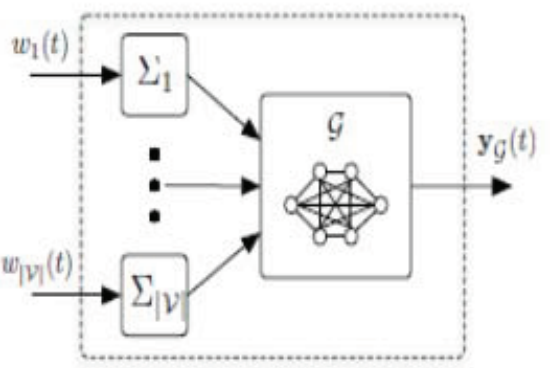

Figure 1: Block diagram of global DNDS

the same set of linear state-space dynamics, that is, $\left\{A_{o i}, \ldots, \Gamma_{o i}\right\},=\left\{A_{o j}, \ldots, \Gamma_{o j}, \forall i, j \in \mathcal{N}\right\}$. Hence, we drop the sub-script for all state-space and operator representations of the system. Using the above notations and the Kronecker properties, we can express the homogeneous dynamics as

$$
\begin{aligned}
\mathbf{x}(k+1) & =\hat{\mathbf{A}} \mathbf{x}(k)+\hat{\mathbf{B}} \mathbf{u}(k)+\hat{\Gamma} \omega(k), \\
\mathbf{z}(k) & =\hat{\mathbf{C}} \mathbf{x}(k)+\hat{\mathbf{D}} \mathbf{u}(k) \\
\mathbf{y}(k) & =\hat{\mathbf{G}} \mathbf{x}(k) \\
\mathbf{y}_{\mathcal{G}}(k) & =\left(\mathcal{E}(\mathcal{G})^{T} \otimes \mathbf{G}\right) \mathbf{x}(k) \\
\hat{\mathbf{A}} & =\left(I_{|\mathcal{V}|} \otimes \mathbf{A}\right), \hat{\mathbf{B}}=\left(I_{|\mathcal{V}|} \otimes \mathbf{B}\right) \\
\hat{\mathbf{\Gamma}} & =\left(I_{|\mathcal{V}|} \otimes \Gamma\right), \hat{\mathbf{G}}=\left(I_{|\mathcal{V}|} \otimes \mathbf{G}\right) \\
\hat{\mathbf{C}} & =\left(I_{|\mathcal{V}|} \otimes \mathbf{C}\right), \hat{\mathbf{D}}=\left(I_{|\mathcal{V}|} \otimes \mathbf{D}\right)
\end{aligned}
$$

2) heterogeneous DNDS, where the dimension of each agent need not be the same. In a similar way and using the Kronecker properties, we can express the heterogeneous dynamics as

$$
\begin{aligned}
\mathbf{x}(k+1) & =\hat{\mathbf{A}} \mathbf{x}(k)+\hat{\mathbf{B}} \mathbf{u}(k)+\hat{\Gamma} \omega(k) \\
\mathbf{z}(k) & =\hat{\mathbf{C}} \mathbf{x}(k)+\hat{\mathbf{D}} \mathbf{u}(k) \\
\mathbf{y}(k) & =\hat{\mathbf{G}} \mathbf{x}(k) \\
\mathbf{y}_{\mathcal{G}}(k) & =\left(\mathcal{E}(\mathcal{G})^{T} \otimes \mathbf{G}\right) \mathbf{x}(k)
\end{aligned}
$$

\section{Static Feedback Control}

In what follows, we present an LMI-based formulation to the static feedback control of system (5) while minimizing the quadratic cost (8).

$$
\mathcal{J}=\sum_{k=0}^{\infty}\left(\mathbf{x}^{T}(k) \mathbf{Q} \mathbf{x}(k)+\mathbf{u}^{T}(k) \mathbf{R u}(k)\right)
$$

We proceed to determine a linear optimal statefeedback control $\mathbf{u}(k)=\mathbf{L x}(k)$ that achieves this goal under the following conditions: 
Assumption 3 System(5)-(6) possesses the following properties:

a. All the states $\mathbf{x}(k)$ are available for feedback,

b. The system a stabilizable which means that all of its unstable modes are controllable,

c. The system are detectable having all its unstable modes observable

We proceed by adopting Lyapunov theory.

Assumption 4 There exists a Lyapunov functional $\mathbf{V}(\mathbf{x}(k))$ which has the properties:

- $\mathbf{V}(\mathbf{x}(k))=\mathbf{x}^{T}(k) \mathbf{P x}(k), \quad \mathbf{P}>0$,

- There exists $\gamma_{+}>0$ such that $\mathbf{x}_{o}^{T}(k) \mathbf{P} \mathbf{x}_{o}(k) \leq$ $\gamma_{+}$

- $\mathbf{V}(\mathbf{x}(k+1))-\mathbf{V}(\mathbf{x}(k)) \leq-\left[\mathbf{x}^{T}(k) \mathbf{Q x}(k)+\right.$ $\left.\mathbf{u}^{T}(k) \mathbf{R u}(k)\right]$

Considering system (5) with linear control $\mathbf{u}(k)=$ $\mathbf{L x}(k)$. The following theorem provides an LMIbased LQR design:

Theorem 5 Given matrices $\mathbf{Q}>0, \mathbf{R}>0$ system (5)-(6) $w(k) \equiv 0$ and the LQR control $\mathbf{u}(k)=\mathbf{L x}(k)$ is asymptotically stable and $J_{\infty} \leq \mathbf{V}\left(x_{0}\right)$ if there exist matrices $\mathbf{X}, \mathbf{Z}>0, \mathbf{W}>0$ such that

$$
\begin{aligned}
& \min _{\gamma_{+}, \mathbf{X}, \mathbf{z}} \gamma_{+} \\
& \text {subject to } \\
& {\left[\begin{array}{ccc}
-\mathbf{X}+\mathbf{W} & \left(\mathbf{X} \hat{\mathbf{A}}^{T}+\mathbf{Z} \hat{\mathbf{B}}^{T}\right) & \mathbf{Z} \\
\bullet & -\mathbf{X} & 0 \\
\bullet & \bullet & -\mathbf{S}
\end{array}\right]<} \\
& {\left[\begin{array}{cc}
\gamma_{+} & x_{o}^{t} \\
\bullet & \mathbf{X}
\end{array}\right] \geq 0}
\end{aligned}
$$

has a feasible solution, then $L Q R$ gain matrix is $\mathbf{L}=$ $\mathbf{S} \mathbf{Y}^{-1}$. the form

Proof: Select Lyapunov functional $\mathbf{V}(\mathbf{x}(k))$ of

$$
\mathbf{V}(\mathbf{x}(k))=\mathbf{x}^{T}(k) \mathbf{P x}(k), \quad \mathbf{P}>0
$$

and satisfies

$$
\begin{aligned}
& \mathbf{V}(\mathbf{x}(k+1))-\mathbf{V}(\mathbf{x}(k)) \leq \\
& -\left(\mathbf{x}^{T}(k) \mathbf{Q} \mathbf{x}(k)+\mathbf{u}^{T}(k) \mathbf{R u}(k)\right)
\end{aligned}
$$

Then, the linear system controlled by $\mathbf{u}(k)$ is asymptotically stable and $\mathcal{J}_{\infty} \leq \mathbf{V}(\mathbf{x}(0))$. With $\mathbf{u}(k)=$ $\mathbf{L X}(k)$, inequality (12) is equivalently expressed as

$$
\begin{aligned}
& \mathbf{x}^{T}(k) \hat{\mathbf{A}}_{L}^{T} \mathbf{P} \hat{\mathbf{A}}_{L} x(k)-\left(\mathbf{x}^{T}(k) \mathbf{P}(\mathbf{x}(k) \leq\right. \\
& -\mathbf{x}^{T}(k)\left[\mathbf{Q}+\mathbf{L}^{T} \mathbf{R} \mathbf{L}\right] \mathbf{x}(k), \\
& \hat{\mathbf{A}}_{L}=\hat{\mathbf{A}}+\hat{\mathbf{B}} \mathbf{L}
\end{aligned}
$$

From (13), it is evident that (12) is satisfied if there exists $\mathbf{L}$ and $\mathbf{P}$ such that

$$
\hat{\mathbf{A}}_{L}^{T} \mathbf{P} \hat{\mathbf{A}}_{L}-\mathbf{P}+\left[\mathbf{Q}+\mathbf{L}^{T} \mathbf{R L}\right]<0
$$

Moreover, instead of directly minimizing the cost $x_{o}^{t} K_{*} x_{o}$, we proceed to minimize its upper bound. Therefore, we assume that there exists $\gamma_{*}>0$ such that

$$
\mathbf{x}^{T}(0) \mathbf{P x}(0) \leq \gamma_{*}
$$

In effect, the linear optimal control problem under consideration for given $\gamma_{*}$ can be cast into the format

$$
\begin{array}{ll} 
& \min _{\gamma_{*}, \mathbf{P}, \mathbf{L}} \gamma_{*} \\
\text { subject to } & (14)-(15)
\end{array}
$$

To convexify the above problem, we first express (14) as

$$
\left[\begin{array}{ccc}
-\mathbf{P}+\mathbf{Q} & \hat{\mathbf{A}}_{L}^{T} & \mathbf{L}^{T} \\
\bullet & \mathbf{P}^{-1} & 0 \\
\bullet & \bullet & \mathbf{R}^{-1}
\end{array}\right]<0
$$

Pre- and post-multiply (17) by $\operatorname{diag}\left\{\mathbf{P}^{-1}, I, I\right\}$ and using $\mathbf{X}=\mathbf{P}^{-1}, \mathbf{W}=\mathbf{X Q X}, \quad \mathbf{Z}=\mathbf{X L}^{T}, \quad \mathbf{S}=$ $\mathbf{R}^{-1}$ it follows that (17) is equivalent to (10). Additionally, inequality (15) can be expressed as inequality (11).

The minimization problem (16) is cast into the form (9)-(11) as desired. When a feasible solution of the convex minimization problem at hand is attained, then we get $\mathbf{P}=\mathbf{X}^{-1} \mathbf{L}=\mathbf{Z}^{T} \mathbf{X}^{-1}$

\section{$5 \quad \mathcal{H}_{2}$ System Norm of DNDS}

We proceed to provide a description of the $\mathcal{H}_{\in}$ system norm by considering the The controllability grammian for an individual agent (from the exogenous input channel) based on the dynamics in (2) is defined as:

$$
\mathcal{X}_{c}^{i}=\sum_{m=o}^{\infty} \mathbf{A}_{i}^{T}{ }^{m} \boldsymbol{\Gamma}_{i}^{T} \boldsymbol{\Gamma}_{i} \mathbf{A}_{i}^{m}
$$


The controllability grammian $\mathcal{X}_{c}^{i}$ can be calculated by solving the corresponding Lyapunov equation

$$
\mathbf{A}_{i} \mathcal{X}_{c}^{i} \mathbf{A}_{i}^{T}-\mathcal{X}_{c}^{i}+\Gamma_{i} \Gamma_{i}^{T}=0
$$

It is known that the $\mathcal{H}_{\in}$ norm of each agent from the exogenous input channel to the measured output can be expressed in terms of the grammian as

$$
\left\|T_{i}^{\omega \rightarrow z}\right\|_{2}=\sqrt{\operatorname{Tr}\left(\mathbf{G}_{i} \mathcal{X}_{c}^{i} \mathbf{G}_{i}^{T}\right)}
$$

where $\operatorname{Tr}($.$) is the trace operator. We proceed to clar-$ ify how the underlying network topology influences the system norm. For simplicity in exposition, we separate our analysis into the homogeneous and heterogeneous cases.

\section{$6 \mathcal{H}_{2}$ Norm of Homogeneous DNDS}

The $\mathcal{H}_{\in}$ norm of the homogeneous DNDS described in (5) can be written in terms of the controllability grammian. We consider the map $T_{h o m}^{\omega \rightarrow \mathcal{G}}$ for examining the global NDS layer. Therefore, the expression for the controllability grammian of the global NDS layer in (5) is

$$
\begin{aligned}
\mathcal{X}_{c} & =\sum_{m=o}^{\infty} \mathbf{A}^{\left(I_{|\mathcal{V}|} \otimes \mathbf{A}\right)} T\left(\mathcal{E}(\mathcal{G})^{T} \otimes \mathbf{G}\right) \mathbf{x} \mathbf{A}^{\left(I_{|\mathcal{V}|} \otimes \mathbf{A}\right)} \\
& =\mathcal{L}(\mathcal{G}) \otimes \mathcal{X}_{c}
\end{aligned}
$$

where $\mathcal{X}_{c}:=\left[\mathcal{X}_{c}^{1}, \ldots, \mathcal{X}_{c}^{N}\right]$ represents the aggregate controllability grammian of agents in the network. On utilizing (20), we have the following characterization of the $\mathcal{H}_{\in}$ norm,

$$
\begin{aligned}
\left\|T_{\text {hom }}^{\omega \rightarrow \mathcal{G}}\right\|_{2} & =\sqrt{\operatorname{Tr}\left(I_{N} \otimes \Gamma\right)^{T} \mathcal{L}(\mathcal{G}) \otimes \mathcal{X}_{c} \operatorname{Tr}\left(I_{N} \otimes \Gamma\right)} \\
& =\|\mathcal{E}(\mathcal{G})\|_{F}\left\|T^{\omega \rightarrow z}\right\|_{2}
\end{aligned}
$$

where $\|R\|_{F}$ denotes the Frobenius norm of the matrix $R$. The expression in (22) gives an explicit characterization of how the network affects the overall gain of the DNDS. In the homogeneous case, we can focus our attention on how the Frobenius norm of the incidence matrix changes with the addition or removal of an edge. Recall that the Frobenius norm of a matrix can be expressed as the sum of the vector 2-norm of each column.

In t he case of the incidence matrix, each column, representing a single edge of the graph, always has the same structure. Therefore, the Frobenius norm of the incidence matrix can be expressed in terms of the number of edges in the graph, $|\mathcal{E}|$, as $\mathcal{E}(\mathcal{G})_{F}=\sqrt{2|\mathcal{E}|}$. It follows that the DNDS $\mathcal{H}_{2}$ norm is only dependent on the number of edges in the graph rather than the actual structure of the topology.
Considering only connected graphs, then we have immediate lower and upper bounds on the $\mathcal{H}_{2}$ norm of the system,

$$
\left\|T_{\text {hom }}^{\omega \rightarrow \mathcal{G}}\right\|_{2}^{2} \geq\left\|\mid T_{\text {hom }}^{\omega \rightarrow y}\right\|_{2}^{2}(|\mathcal{V}|-1)
$$

The lower bound is attained with equality whenever the underlying graph is a spanning tree. Assuming that all graphs are simple, that is they do not have multiple edges between a single pair of nodes, then the upper bound for the system norm is achieved by the complete graph,

$$
\left\|T_{\text {hom }}^{\omega \rightarrow \mathcal{G}}\right\|_{2}^{2} \leq\left\|\left|T_{\text {hom }}^{\omega \rightarrow y} \|_{2}^{2}\right| \mathcal{V} \mid(|\mathcal{V}|-1)\right.
$$

\section{$7 \quad \mathcal{H}_{2}$ Norm of Heterogeneous DNDS}

In this case, the DNDS $\mathcal{H}_{\in}$ norm can be established in view of (20) as

$$
\begin{aligned}
& \left\|T_{\text {het }}^{\omega \rightarrow \mathcal{G}}\right\|_{2}^{2}= \\
& \operatorname{Tr}\left\{\left(\otimes I_{N} \mathcal{E}(\mathcal{G})^{T}\right) \mathbf{G} \mathcal{X}_{c} \mathbf{G}^{T}\left(\otimes I_{N} \mathcal{E}(\mathcal{G})\right)\right\}
\end{aligned}
$$

where $\mathcal{X}_{c}$ denotes the block diagonal aggregate of the controllability grammian of the agents as introduced in (18). It is crucial to note that $\operatorname{Tr}\left\{\mathbf{G} \mathcal{X}_{c} \mathbf{G}^{T}\right\}=$ $\sum_{i=1}^{|\mathcal{V}|}\left\|T_{i}^{\omega \rightarrow y}\right\|_{2}^{2}$. Invoking cycle property of the trace operator and exploiting the block diagonal structure of the argument simplifies into

$$
\begin{aligned}
& \operatorname{Tr}\left\{\mathbf{G} \mathcal{X}_{c} \mathbf{G}^{T}\left(\Delta(\mathcal{G})-A(\mathcal{G}) \otimes I_{N}\right)\right\}= \\
& \sum_{i=1}^{|\mathcal{V}|} \operatorname{Tr}\left\{\mathbf{G} \mathcal{X}_{c} \mathbf{G}^{T}\left(d_{i} \otimes I_{N}\right)\right\}=\sum_{i=1}^{|\mathcal{V}|} d_{i}\left\|T_{i}^{\omega \rightarrow y}\right\|_{2}^{2}(26)
\end{aligned}
$$

where $d_{i}$ is the degree of the $i^{t h}$ agent in the graph. In turn, this can now be used to obtain the following expression for the $\mathcal{H}_{2}$ norm of the system,

$$
\left\|T_{\text {het }}^{\omega \rightarrow \mathcal{G}}\right\|_{2}=\sqrt{\left.\sum_{i=1}^{|\mathcal{V}|} d_{i}\left\|T_{i}^{\omega \rightarrow y}\right\|_{2}^{2}\right)}
$$

This eventually leads to

$$
\begin{aligned}
& \left\|T_{\text {het }}^{\omega \rightarrow \mathcal{G}}\right\|_{2}= \\
& \left\|\left[\begin{array}{lll}
\left\|T_{1}^{\omega \rightarrow y}\right\|_{2} & & \\
& \ddots & \\
& & \left\|T_{|\mathcal{V}|}^{\omega \rightarrow y}\right\|_{2}
\end{array}\right] \mathcal{E}(\mathcal{G})\right\|_{F}
\end{aligned}
$$

It is significant to observe that when each agent has the same dynamics, (28) reduces to the expression in (22). This characterization discloses an interesting feature of how the placement of an agent within a certain topology affects the overall system gain. In order to minimize the gain, it is beneficial to keep systems with high norm in locations with minimum degree. 


\section{Conclusion}

This paper focused on the analysis of a class of linear DNDS based on a relative sensing model. The ensuing results disclose an important connection between certain graph-theoretic concepts and systemstheoretic properties. A new state-feedback agent controller is derived, the gain of which by linear matrix inequality. Some salient features pertain to the application of convex optimization for designing interconnection topology for overall optimal $\mathcal{H}_{2}$ performance. This paper also illuminates a close relationship between systems-theoretic properties and graph properties in DNDS, which can be exploited for synthesis of controllers with prescribed criteria. This methodology can be easily extended in 2-D Systems.

Acknowledgments This work is supported by the deanship of scientific research (DSR) at KFUPM for support through distinguished professorship award project no. (IN 161065).

\section{References:}

[1] W. Ren and R. W. Beard, "A survey of consensus problems in multiagent coordination", Proc. $A C C$, Portland, OR, 2005.

[2] M. Mesbahi and F. Y. Hadaegh, "Formation flying control of multiple spacecraft via graphs, matrix inequalities, and switching", Proc. AIAA $J G C D$, vol. 24, no. 2, pp. 369-377, 2001.

[3] M. S. Mahmoud, "Networked control systems analysis and design: An overview", The Arabian Journal of Science and Engineering: Invited Paper, vol. 41, no. 3, pp. 711-758, March 2016.

[4] P. K. C. Wang and F. Y. Hadaegh, "Coordination and control of multiple micro-spacecraft moving in formation", J. Astronaut. Sci., vol. 44, pp. 315-355, 1996.

[5] J. A. Fax and R. M. Murray, "Information flow and cooperative control of vehicle formations", IEEE Trans. Automatic Control, vol. 49, no. 9, pp. 1465-1476, 2004.

[6] A. Rahmani and M. Mesbahi, "Pulling the strings on agreement: anchoring, controllability, and graph automorphisms, ACC, NY, 2007.

[7] D. Zelazo and M. Mesbahi, "On the observability properties of homogeneous and heterogeneous networked dynamic systems", IEEE CDC, Cancun, Mexico, 2008.

[8] R. S. Smith and F. Y. Hadaegh, "Control of deep space formation flying spacecraft; relative sensing and switched information", AIAA JGCD, vol. 28 , no. $1,2005$.

[9] C. Godsil and G. Royle, Algebraic Graph Theory, Springer, 2001.
[10] R. A. Horn and C. Johnson, Topics in Matrix Analysis, Cambridge University Press, 1991.

[11] M. S. Mahmoud, Advanced Control Design with Application to Electromechanical Systems, Elsevier Butterworth-Heinemann, Brazil, April 2018.

[12] B. Korte and J. Vygen,Combinatorial Optimization: Theory and Algorithms, Springer-Verlag, Berlin Heidelberg, 2000.

[13] C. R. Johnson, Matrix Theory and Applications, Proc. Symposia in Applied Mathematics (40), 1990.

[14] F. Bullo, J. Cortes, and S. Martinez, Distributed Control of Robotic Networks: a Mathematical Approach to Motion Coordination Algorithms, Princeton, NJ: Princeton University Press, 2009.

[15] M. Mesbahi and M. Egerstedt, Graph Theoretic Methods in Multiagent Networks, Princeton, NJ: Princeton University Press, 2010. 